\title{
Cellular locations of Proteinases and Association with VESICLES IN PORPHYROMONAS GINGIVALIS
}

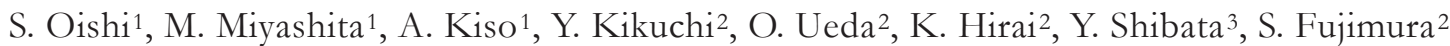 \\ ${ }^{1}$ Department of Oral Health Promotion, Graduate School of Oral Medicine, \\ ${ }^{2}$ Department of Oral Microbiology, \\ ${ }^{3}$ Division of Oral Health Promotion, Institute for Oral Science, \\ Matsumoto Dental University, Shiojiri-Nagano, Japan
}

\begin{abstract}
We found that locations of arginine-specific gingipain (RGP) in the cellular fractions in the crude extract, envelope, vesicles, and culture supernatants were $48 \%$, $16 \%, 17 \%$, and $31 \%$, respectively, and the corresponding values of lysine-specific gingipain (KGP) were $47 \%, 10 \%, 7 \%$, and $36 \%$, respectively. Although the molecular mass of RGP in the culture supernatant had been determined as $43 \mathrm{kDa}$, and that of KGP had been as $48 \mathrm{kDa}$, molecular masses of both proteinases solubilized from the vesicles were estimated to be over $1,500 \mathrm{kDa}$, since they eluted in the void volume of the column in the gel filtration on Sephacryl S-300. There was no reduction of molecular size by the following treatment with SDS, high-concentration $\mathrm{NaCl}$, or urea. Interestingly, the occurrence of the macromolecular forms could not observed in other enzymes tested such as monopeptidyl, dipeptidyl, and tripeptidyl peptidases, as well as alkaline phosphatase. Therefore, occurrence of the macromolecular forms may be restricted to the proteinases. When the vesicle and culture supernatants containing free RGP and KGP were mixed and incubated, neither RGP nor KGP seemed to bind to vesicles. RGP bound to the vesicle was found to be more stable to heat treatment than the free form, suggesting that association of RGP with the vesicle caused heat stability of this enzyme.
\end{abstract}

Key words: proteinase, enzyme, RGP, KGP, P. gingivalis, vesicle

\section{INTRODUCTION}

Gram-negative, black-pigmented obligatory anaerobes including Porphyromonas gingivalis, Prevotella intermedia, and Prevotella nigrescens have been implicated as etiological agents of human periodontitis, of which $P$. gingivalis is the most potent pathogen of this disease $[1,2,3,4]$. RGP hydrolyses the peptide bonds of arginine-Xa.a. and KGP splits that of lysine-Ya.a., both are major proteinases of $P$. gingivalis, and these enzymes are considered to be important in the pathogenesis of periodontitis. The biochemical properties of these enzymes were described in the last decade and proteolytic enzymes have been implicated as important pathogenic factors $[5,6,7]$. However, obser- vations from the biological aspects remain unsatisfactory.

Vesicles have been thought to originate from the outer membranes of gram-negative bacterial species. Toxic substances were found in the vesicles of Aggregatibacter (Actinobacillus) actinomycetemcomitans and they were considered to be released into the crevicular environment, which may contribute to the pathogenesis of this species [8, 9]. Grenier and Mayrand described the vesicles of Porphyromonas gingivalis in which they reported that vesicles of approximately 50 $\mathrm{nm}$ predominated and contained highly active enzymes against collagen, Azocoll, and a synthetic substrate of RGP. They also established methods of vesicle preparation from liquid cultures [10].

Vesicles of $P$. gingivalis from the outer membrane were prepared by a combination of mechanical disruption of the cells followed by $80,000 \mathrm{xg}$ centrifugation and sarkosyl treatment of the cells, sonication, and $100,000 \mathrm{xg}$ centrifugation. Extracellular vesicles were obtained by precipitation with ammonium sulfate from culture supernatant [11]. Sarkosyl-insoluble preparation and extracellular vesicles yielded similar protein patterns in SDS-PAGE. However, vesicles prepared directly from the cells contained additional proteins. The mechanism of formation and release of theses vesicles in $P$. gingivalis was proposed as a result of "herniation" of the outer membrane in the process of turnover of peptidoglycan [12].

We noticed that RGP and KGP are found in the culture supernatant, in the surfaces and within the cytoplasm. As for those in the culture supernatant, two types of enzymes exist: one is a free form and the other is bound to released vesicles. The free forms of RGP and KGP were isolated and their molecular masses were determined to be $43 \mathrm{kDa}$ and $48 \mathrm{kDa}$, respectively $[13,14]$. Since vesicles of gram-negative bacteria may play an important role in the transportation of their virulence factors to the host cells [9], we attempted to undertake an investigation of the interaction of proteinases and the vesicles.

\section{Materials AND METHOds}

All procedures were conducted at $4{ }^{\circ} \mathrm{C}$, if not otherwise specified. 


\section{BACTERIAL STRAINS AND CULTIVATION}

P. gingivalis ATCC 33277 was used mainly in this study although $P$. gingivalis W83 and 381 were also employed. These strains were maintained anaerobically at $37{ }^{\circ} \mathrm{C}$ on blood agar plates containing hemin (5 $\mu \mathrm{g} / \mathrm{ml})$ and menadione $(0.5 \mu \mathrm{g} / \mathrm{ml})$. The fresh cultures of the strains were inoculated into a medium containing Trypticase peptone (17 g/liter), yeast extract (3 $\mathrm{g} /$ liter $), \mathrm{NaCl}$ (5 g/liter), $\mathrm{K}_{2} \mathrm{HPO}_{4}(2.5 \mathrm{~g} /$ liter $)$, hemin (5 mg/liter), and menadione $(0.5 \mathrm{mg} /$ liter $)$ and cultured at $37^{\circ} \mathrm{C}$ in a glove box filled with a mixture of gases $\left(\mathrm{N}_{2}: \mathrm{H}_{2}: \mathrm{CO}_{2} ; 85: 10: 5\right)$ for 2 days.

\section{Preparation of Bacterial Fractions}

Culture supernatant containing vesicles were prepared from the whole culture by centrifugation at 10,000 xg for $15 \mathrm{~min}$. Vesicles were collected by the methods described by Grenier et al. [10] with minor modifications: ammonium sulfate was added to the culture supernatant at a concentration of $40 \%$ saturation of this reagent and stirred for $5 \mathrm{~h}$. The mixtures were centrifuged at 25,000 $\mathrm{xg}$ for $20 \mathrm{~min}$, and the precipitate was suspended in $50 \mathrm{mM}$ Tris-HCl buffer, $\mathrm{pH} 8.2$ and dialyzed against the same buffer. After dialysis, the suspension was centrifuged at 40,000 $\mathrm{xg}$ for $30 \mathrm{~min}$ and the precipitate was referred to as the vesicle fraction. The concentration of the supernatant of the $40 \%$ saturation of ammonium sulfate mixture was raised to $75 \%$ saturation; the precipitate was collected by centrifugation and dissolved in $50 \mathrm{mM}$ Tris- $\mathrm{HCl}$ buffer, $\mathrm{pH}$ 8.2, followed by dialysis against the same buffer. This fraction was designated the concentrated culture supernatant. Analytical size of culture supernatant free from particles was prepared from the vesicle containing culture supernatant $(10,000 \mathrm{xg}$ supernatant of whole culture) by centrifugation at 100,000 $\mathrm{xg}$ for $60 \mathrm{~min}$. On the other hand, cells were disrupted by sonication at $150 \mathrm{~W}$ for 20 min. Unbroken cells and debris in the sonicate were removed by centrifugation at $6,000 \mathrm{xg}$ for $15 \mathrm{~min}$, then the supernatant was centrifuged once more at 100,000 xg for $60 \mathrm{~min}$. The resultant supernatant was designated crude extract and the precipitate was the envelope.

\section{Solubilization of Vesicles and EnVElope by TRITON X-100}

Triton X-100 was added to $0.5 \%$ to the suspensions of the vesicles and the envelope in $50 \mathrm{mM}$ Tris- $\mathrm{HCl}$ buffer ( $\mathrm{pH}$ 8.2) and stirred for $30 \mathrm{~min}$ at room temperature, followed by centrifugation at 100,000 $\mathrm{xg}$ for $1 \mathrm{~h}$. The supernatant solutions were stored as solubilized fractions of vesicles and envelope.

\section{DETERMinATION OF ENZYME}

Activities of arginine-specific proteinase (RGP) and lysine-specific proteinase (KGP) were estimated using chromogenic synthesized substrates, benzoylargininep-nitroanilide and tosyl-glycylprolyllysine-p-nitroanilide, respectively, accordingly our previous reports [13, 14]. Dipeptidyl peptidase activities were assayed using $p$-ni- troanilide derivatives of glycyl-phenylalanine (DPP 1), lysyl-alanine (DPP 2), glycyl-proline (DPP 4). Proline specific tripeptidyl peptidase activity was determined with alanyl-alanyl-proline- $p$-nitroanilide. Alkaline phosphatase activity was measured photometrically using $p$ nitrophenylphosphate [15].

\section{Purification of RGP of Culture Supernatant}

Free form $43 \mathrm{kDa}$ RGP and $48 \mathrm{kDa}$ KGP of $P$. gingivalis ATCC 33277 in the culture supernatant were purified by the procedures including concentration by ammonium sulfate, ion-exchange chromatography, gel filtration, and isoelectric focusing $[13,14]$.

\section{Partial Purification of Vesicle-Bound RGP AND KGP}

The solubilized materials of vesicle were applied to a column of Q-Sepharose (GE Healthcare UK Ltd) equilibrated with $50 \mathrm{mM}$ Tris- $\mathrm{HCl}$ buffer, $\mathrm{pH}$ 8.5. After the column was washed with the same buffer, the proteins were eluted with a linear gradient of $\mathrm{NaCl}$ from 0 to $800 \mathrm{mM}$. The active fractions were concentrated and dialyzed against $50 \mathrm{mM}$ Tris- $\mathrm{HCl}$ buffer, $\mathrm{pH} 8.0$ containing $200 \mathrm{mM} \mathrm{NaCl}$ and subjected to gel filtration on Sephacryl S-300 HR (GE Healthcare) and eluted with this buffer saline. The eluates in the void volume of the column containing RGP and KGP activities were collected and designated partially purified RGP and KGP.

\section{Gel Filtration}

Recovered RGP and KGP activities from vesicle by Triton X-100 treatment were $72 \%$ and $68 \%$, respectively. Sephacryl S-300 HR packed in a column $(2.6 \mathrm{~cm}$ by $94 \mathrm{~cm}$ ) was used for gel filtration of the solubilized materials. The column was eluted at a flow rate of 40 $\mathrm{ml} / \mathrm{h}$ with $50 \mathrm{mM}$ Tris-HCl buffer, $\mathrm{pH} 8.0$ containing $200 \mathrm{mM} \mathrm{NaCl}$, if not otherwise stated. The samples to be subjected were dialyzed against this buffer saline before application to columns.

\section{Treatment of Solubilized Materials of Vesicles by SEVERAL REAGENTS AND GEL FiLTRATION}

Solubilized materials of vesicles were mixed separately with the following reagents, $\mathrm{NaCl}(0.5 \mathrm{M})$, SDS (1\%), dithiothreitol $(5 \mathrm{mM})$, and urea $(6 \mathrm{M})$ in $50 \mathrm{mM}$ Tris$\mathrm{HCl}$ buffer, $\mathrm{pH} 8.0$ and incubated at room temperature for $45 \mathrm{~min}$. After the incubation, each mixture was applied to a column of Sephacryl S-300 HR (2.6 by $94 \mathrm{~cm}$ ) which had been equilibrated with $50 \mathrm{mM}$ Tris-HCl buffer, pH 8.0 containing each reagent and eluted with the same corresponding buffer.

\section{RESULTS}

\section{LOCATIONS OF RGP AND KGP}

Yields of vesicle and envelope, and profiles of the cellular locations of RGP and KGP in culture supernatant, crude extract, vesicle, and envelope of three 
Table 1. Cellular locations of RGP and KGP.

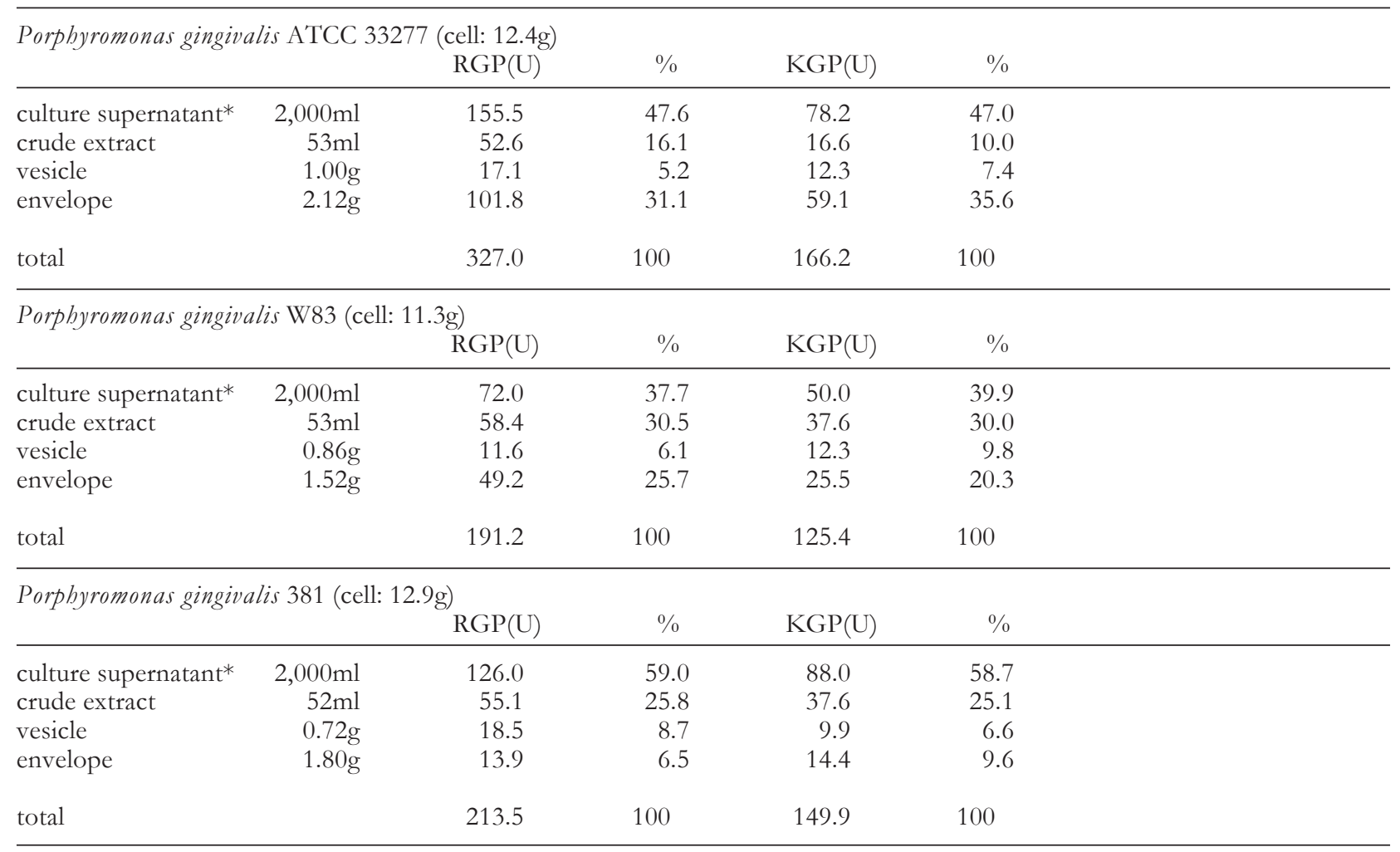

*; particle free

strains of $P$. gingivalis are summarized in Table 1 . Although the bacteria were found to grow as the same level, slight differences were observed in the yields of vesicle and envelope. RGP and KGP were detected in all fractions, and production of RGP appeared to exceed that of KGP in the three strains. The fraction containing the maximum amounts of these proteinases was the culture supernatants in all the three strains. Distribution of RGP and KGP in the four fractions were quantitatively similar, except the lower amounts of both proteinases in the envelope of strain 381 .

\section{Gel Filtration Profiles of Proteinases and OTHER ENZYMES}

The purified RGP of culture supernatant $(43 \mathrm{kDa})$ eluted at elution volume of $335 \mathrm{ml}$ in the gel filtration on Sephacryl S-300 (Fig. 1); however, the RGP solubilized from vesicles eluted in the void volume of the column $(205 \mathrm{ml})$ with a negligible peak of the activity at the corresponding position of the purified culture supernatant RGP (Fig. 2). These findings indicate that the molecular mass of RGP originated from the vesi-

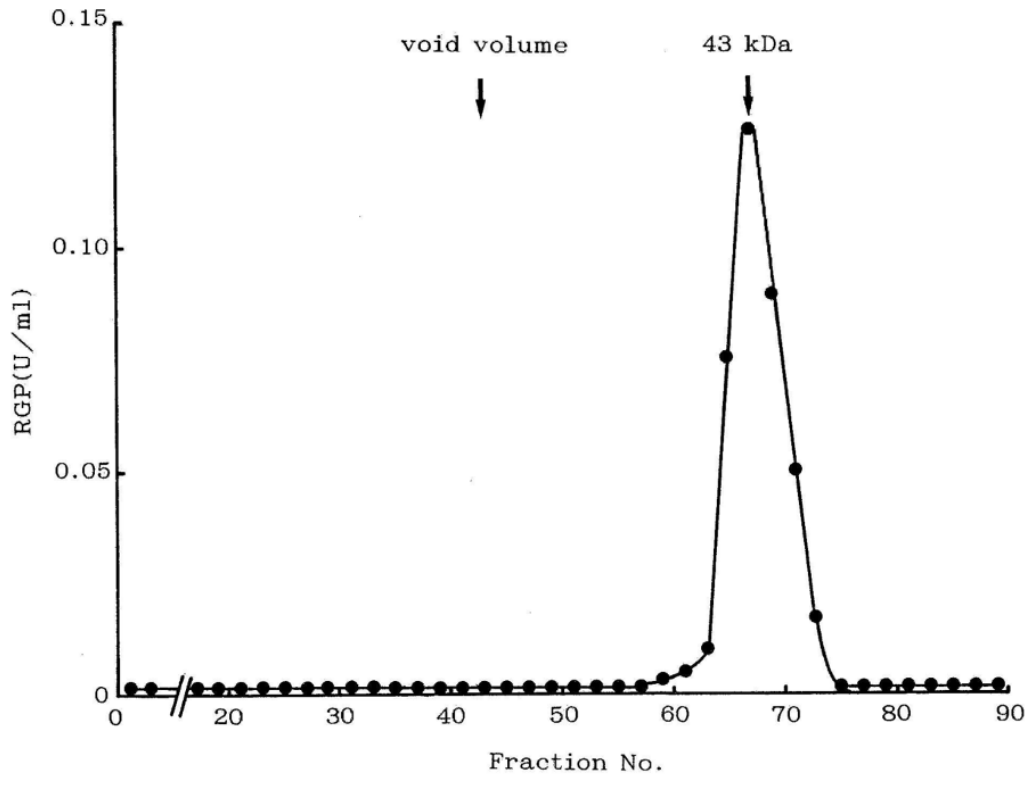

Fig. 1. Gel filtration on Sephacryl S-300 of RGP purified from culture supernatant. The column $(2.6$ by $94 \mathrm{~cm}$ ) was eluted with $50 \mathrm{mM}$ Tris-HCl, pH 8.0 containing $200 \mathrm{mM} \mathrm{NaCl}$. 


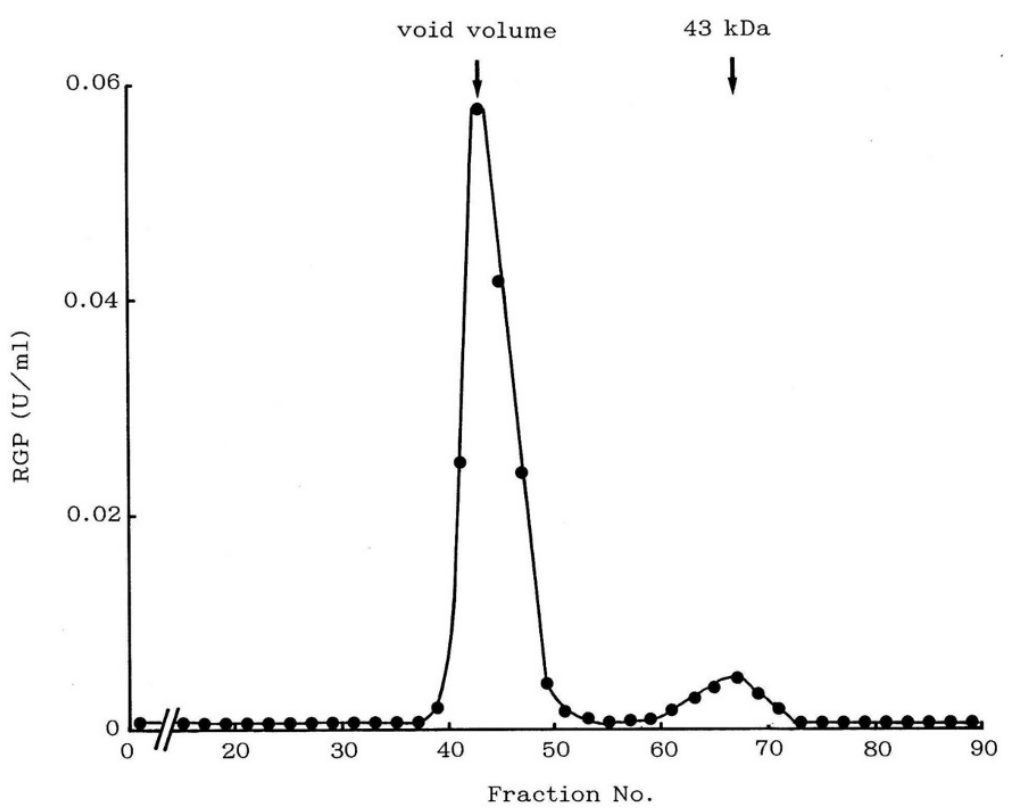

Fig. 2. Gel filtration on Sephacryl S-300 of RGP partially purified from the vesicle. The column $(2.6$ by $94 \mathrm{~cm})$ was eluted with $50 \mathrm{mM}$ Tris-HCl, pH 8.0 containing $200 \mathrm{mM} \mathrm{NaCl}$.
Table 2. Elution positions of RGP, KGP, and some other enzymes in the gel filtration on Sephacryl S-300.

void volume valid volume

\section{RGP}

crude extract

culture supernatant

vesicle extract

envelope extract

KGP

crude extract

culture supernatant

vesicle extract

envelope extract

Glycyl-phenylalaninyl-pNA (DPP 1)

crude extract

culture supernatant

vesicle extract

envelope extract

Lysyl-alanyl-pNA (DPP 2)

crude extract

culture supernatant

vesicle extract

envelope extract

Glycyl-prolyl-pNA (DPP 4)

crude extract

culture supernatant

vesicle extract

envelope extract

$\begin{array}{ll}+ & + \\ - & + \\ + & \text { t } \\ + & +\end{array}$

+
+
tr
+

Alanyl-alanyl-prolyl-pNA

crude extract

culture supernatant

vesicle extract

envelope extract

Alkaline phosphatase

crude extract

culture supernatant

vesicle extract

envelope extract cles may be over $1,500 \mathrm{kDa}$ (by the manual of Sephacryl S series). RGP eluted at the void volume was temporarily referred to as macromolecular form RGP. Gel filtration profiles of KGP were substantially the same as those of RGP; however, significant amounts of activity in vesicle sample were detected in the valid volume, and the approximate ratio of the activities in the void volume and to the valid volume was 2 to 1 . To examine whether $43 \mathrm{kDa}$ RGP was able to convert to the macromolecular form simply by incubation with vesicles, mixtures of vesicle and the purified $43 \mathrm{kDa}$ or raw RGP (concentrated culture supernatant) were subjected to gel filtration, but no shift of the elution volume of $43 \mathrm{kDa}$ RGP to the void volume was seen.

Efforts were made to assess the gel filtration profiles of other enzymes. As shown in Table 2, no activities were detected in the void volume position in the tested enzymes such as dipeptidyl peptidases (DPP-1, DPP-2, and DPP-4), tripeptidyl peptidase, and alkaline phosphatase. Theses were found in single peaks of the activities at the individual specified positions in the valid volume of the column, but not in the void volume.

Treatment of RGP solubilized from vesicles with $\mathrm{NaCl}$ (0.5 M), SDS (1\%), dithiothreitol (5 mM), and urea $(6 \mathrm{M})$ caused no effect on elution profiles of gel filtration. Activities in all cases were detected in the void volume, but not in the valid volume of the column of Sephacryl S-300 HR.

\section{Comparisons of Heat Stability and Sensitivity TO ENZyme InHibitors of FreE AND Vesicle- BOUND RGP AND KGP}

When the purified $43 \mathrm{kDa}$ RGP was heated at $60{ }^{\circ} \mathrm{C}$ for $10 \mathrm{~min}$, the residual activity was only $8.5 \%$ of the control unheated sample; however, the extracted RGP solubilized from the vesicle by detergent maintained $43.5 \%$ of the control activity. Whereas, both samples of KGP solubilized from vesicle and purified from

tr; trace 
culture supernatant were completely inactivated by this heat treatment. No different sensitivity to the RGP inhibitors including EDTA, tosyl-L-lysinechloromethyl ketone, antipain, leupeptin, p-hydroxymercuribenzoate, and L-trans-Epoxy-succinylleucylamido-(4-guanidino)butane was observed between the two types of RGPs. Difference of activation rate by glycyl-glycine was also not noticed.

\section{DISCUSSION}

Proteolytic enzymes are considered to be important etiological factors in periodontitis; however, transportation mechanisms of the bacterial virulent substances to the host mucosa should also be noticed. Since vesicles are considered to play a vehicular role $[8,9]$, we tried to evaluate the presence of RGP and KGP in vesicles, as well as in other subcellular fractions and the surrounding culture medium. We confirmed that vesicles contained RGP and KGP, even if both enzymes accounted for only small rates in the subcellular fractions (Tab1e 1).

Fujimura et al. reported that in the early stage of cultivation of Staphylococcus aureus, the molecular mass of staphylokinase in the culture supernatant was determined by gel filtration as $15 \mathrm{kDa}$ (Type A); however, in the late stage, it appeared to convert to 320 $\mathrm{kDa}$ (Type B), indicating that Type B staphylokinase might be a complex of some high-molecular size substance. This complex could be dissociated by treatment with high concentrations of $\mathrm{KCl}$ or detergent [16]. However, formation of macromolecular mass RGP described in this report could be caused in a different manner from the conversion of molecular size of staphylokinase. We confirmed that RGP solubilized from the vesicles taken in the early stage of cultivation $(15 \mathrm{~h})$ also was expelled from the molecular sieving on Sephacryl S-300 (data not shown).

No difference in effect of inhibitors and activators on the free form and vesicle-bound form of RGP was observed, but a significant difference of thermostability was seen in these two forms of RGP. In other words, vesicles contributed to the thermostability of RGP. The reason why the vesicle-bound RGP was more stable in the heat treatment than free RGP remains unclear. It is also unexplained why the effect of vesicle on the thermostability was not observed in KGP. However, it may be related to the observations that envelope-associated RGPs were found to be more stable than KGP (17).

Recently, $P$. gingivalis outer membrane vesicles were observed entering HeLa cells and surviving within the endocytic organelles (18). We attempted also to evaluate the binding of vesicles to mammalian cells using human epithelial cell A549, cultured in Dullbecco's modified Eagle's medium supplemented with 10\% fetal bovine serum (FBS) under $5 \% \mathrm{CO}_{2}$ for 7 days and red blood cell of horse, sheep, rabbit, and human. Binding was assessed by reduction of RGP activity associated to the vesicles in the centrifugal supernatants at $1,000 \mathrm{xg}$ of the reaction mixtures of cell suspensions and vesicles, based on the previous observation that these cells are precipitated by low speed centrifugation, but that vesicles remained in the supernatant.
However, we could confirm significant binding of the vesicles neither to epithelial cells nor to red blood cells.

\section{REFERENCES}

1. Mayrand D, Holt SC (1988) Biology of assacharolytic black-pigmented Bacteroides species. Microbiol Rev 52: 134-152

2. Slots J, Genco RJ (1984) Black-pigmented Bacteroides species, Capnocytophaga species, and Actinobacillus actinomycetemcomitans in human periodontal disease: virulence factors in colonization, survival, and tissue destruction. J Dent Res 63:412-421

3. Slots J, Listgarten, MA (1988) Bacteroides gingivalis, Bacteroides intermedius, and Actinobacillus actinomycetemcomitans in human periodontal diseases. J Clin Periodontol 15:85-93

4. Van Winkelhoff AJ, Van Steenbergen TJM, De Graaff J (1988) The role of black-pigmented Bacteroides in human oral infections. J Clin Periodontol 15:145-155

5. Grenier D, Mayrand D (1987) Selected characteristics of pathogenic and nonpathogenic strains of Bacteroides gingivalis. J Clin Microbiol 25:738-740

6. Marsch PD, McKee AS, McDermid AS, Dowsett AB (1989) Ultrastructure and enzyme activities of a virulent and an avirulent variant of Bacteroides gingivalis W50. FEMS Microbiol Lett 59:181-186

7. Smalley JW, Birss AJ, Kay HM, McKee AS Marsh PD (1989) The distribution of trypsin-like enzyme activity in cultures of a virulent and an avirulent strain of $\mathrm{Bac}$ teroides gingivalis W50. Oral Microbiol Immunol 4:178179

8. Hammond BC, Stevens RH (1982) Capnocytophaga and Actinobacillus actinomycetemcomitans: occurrence and pathogenic potential in juvenile periodontitis. p. 46-61 In: Genco RJ, Mergenhagen SE (ed.), Host-parasite interactions in periodontal disease. American Society of Microbiology, Washington, D.C.

9. Nowotny A, Behling UH, Hammond B, Lai, C-H, Listgarten M, Pham PH, Sanavi F (1982) Release of toxic vesicles by Actinobacillus actinomycetemcomitans. Infect Immun 37:151-154

10. Grenier D, Mayrand D (1987) Functional characterization of extracellular vesicles produced by Bacteroides gingivalis. Infect Immun 55:111-117

11. Deslauriers M, ni Eiden Deirdre, Lamonde L, Mouton C (1990) SDS-PAGE analysis of protein and lipopolysaccharide of extracellular vesicules and Sarkosyl-insoluble membranes from Bacteroides gingivalis. Oral Microbiol Immunol 5:1-7

12. Zhou L, Srisatjaluk, Justus DE, Doyle R J (1998) On the origin of membrane vesicles in Gram-negative bacteria. FEMS Microbiol Lett 163:223-228

13. Fujimura S, Nakamura T (1990) Purification and characterization of a $43-\mathrm{kDa}$ protease of Bacteroides gingivalis. Oral Microbiol Immunol 5:360-362

14. Fujimura S, Shibata Y, Nakamura T (1993) Purification and partial characterization of a lysine-specific protease of Porphyromonas gingivalis. FEMS Microbiol Lett 113: 133138

15. Torriani A (1968) Alkaline phosphatase of Escherichia coli. Methods in Enzymology 12B:212-218

16. Fujimura S, Makino T, Hayashi TTA (1974) Occurrence of a complex form of staphylokinase in the course of cultivation of Staphylococcus aureus. Appl Microbiol 28:5-10

17. Fujimura S, Hirai K, Shibata Y, Nakayama K, Nakamura $\mathrm{T}$ (1998) Comparative properties of envelope-associated arginine-gingipains and lysine-gingipain of Porphyromonas gingivalis. FEMS Microbiol Lett 163:173-179. 
18. Furuta N, Tsuda K, Omori H, Yoshimori T, Yoshimura F, Amano A (2009) Porpbyromonas gingivalis outer membrane vesicles enter human epithelial cells via an endocytic pathway and sorted to lysosomal compartments. Infect Immun 77:4187-4196

Received: January 11, 2010 / Accepted: May 21, 2010
Address for correspondence:

Setsuo Fujimura, D.Sc.

Department of Oral Microbiology

Matsumoto Dental University

1780 Hirooka-Gobara

Shiojiri-Shi, Nagano-Ken

399-0781 Japan

Phone/Fax: +81(263)51-2084

E-mail: $\quad$ fujimura@po.mdu.ac.jp 